\title{
Desastres naturales y catastrofismo en el siglo XVIII
}

\author{
Jorge Ordaz \\ Universidad de Oviedo
}

Resumen

En este trabajo se repasan algunos de los desastres naturales más importantes y de mayor repercusión acaecidos en el siglo XVIII en España y en el resto del mundo. Siguiendo la clasificación establecida para los riesgos naturales, el artículo define y sistematiza los diferentes tipos de catástrofes naturales, centrándose especialmente en las de carácter geológico y geoclimático. Así, por ejemplo, dentro de estos tipos de desastres se revisan terremotos, erupciones volcánicas, inundaciones y movimientos de ladera. Especial hincapié se hace en el terremoto de Lisboa de 1755, cuya extraordinaria intensidad y fatales consecuencias suscitaron polémicas científicas, contribuyendo de este modo al interés por el estudio de los seísmos y al avance en el conocimiento de sus causas. Finalmente se concluye con una referencia al debate mantenido, a finales del siglo XVIII y principios del siguiente, entre catastrofistas y gradualistas, y su incidencia en la evolución y desarrollo de las ideas geológicas modernas.

\section{Introducción}

A lo largo de los siglos, el hombre ha tenido que afrontar catástrofes de todo tipo. En los relatos y crónicas de antiguas civilizaciones se habla ya de grandes desastres naturales. La existencia, por ejemplo, de una inundación de enormes proporciones se encuentra en leyendas y mitos primigenios de numerosas 
culturas. Así consta, entre otros textos, en la epopeya de Gilgamesh, escrita hacia el 2600 a.de C. Dicha tradición, presumiblemente basada en un hecho real, sería recogida igualmente por la Biblia en la historia de Noé y el diluvio universal ${ }^{1}$. Otros desastres son mencionados en las Sagradas Escrituras, tales como las plagas de Egipto o la destrucción de Sodoma y Gomorra, cuyo recuerdo ha alimentado la memoria colectiva de muchos pueblos de tradición judeocristiana. Más cercana en el tiempo, la explosión volcánica de la isla de Santorini (actual Thera) en el mar Egeo, en 628 a.C., parece estar, según algunos autores, en el origen del mito de la Atlántida que describiera Platón, así como en el inicio del declive de la civilización minoica ${ }^{2}$. Impactante debió de resultar asimismo la destrucción, el año 79 de nuestra era, de las ciudades de Pompeya y Herculano bajo una lluvia de cenizas procedentes del Vesubio. Ya en tiempos más recientes, el registro histórico de terremotos, erupciones volcánicas, inundaciones y otras catástrofes, se hace cada vez más abundante ${ }^{3}$.

En España se han producido a lo largo del tiempo numerosos desastres naturales. De algunos de ellos se guarda aún memoria; de otros, sin embargo, apenas quedan huellas. Este artículo se centra en algunos de los principales desastres naturales acaecidos en España y en el mundo en el siglo XVIII, haciendo especial hincapié en el terremoto de Lisboa de 1755. Finalmente se concluye con un breve comentario acerca de las ideas catastrofistas y su incidencia en el desarrollo de las ciencias geológicas.

\section{Riesgos naturales: concepto y tipos}

Con anterioridad a una catástrofe siempre existe el riesgo de que se produzca. Si hablamos de riesgos naturales éstos se hallan repartidos por todo el planeta y son inherentes al mismo. Los riesgos naturales se suelen dividir en dos grandes apartados: biológicos y físicos. Entre los primeros figuran toda clase de plagas y epidemias. Los físicos se subdividen, a su vez, en cuatro grupos: los cósmicos, los climáticos, los geológicos y los geoclimáticos. Son éstos dos últimos grupos los que reúnen algunos de los más frecuentes y peligrosos desastres naturales y de los que se hablará aquí. Por lo que se refiere a los riesgos geológicos, no existe una definición universalmente aceptada. Una acepción amplia sería la siguiente: «Todo proceso, situación o suceso en el medio geológico, natural, inducido o mixto, que puede generar un daño económico o

\footnotetext{
${ }^{1}$ Un repaso a las grandes inundaciones históricas y sus efectos puede verse en MiLne, 1986.

${ }^{2}$ Consúltese Galanopoulos y Bacon, 1969.

${ }^{3}$ Consúltese, p. ej., Davis, 1991.
} 
social para alguna comunidad, y en cuya predicción, prevención o corrección han de emplearse criterios geológicos» ${ }^{4}$. Según esta definición los riesgos geológicos pueden clasificarse, de acuerdo con su origen, en: naturales; es decir, ligados a procesos geodinámicos internos (volcanes, terremotos) o externos (subsidencias $^{5}$, movimientos de ladera); inducidos (p. ej. por rotura de presas); y mixtos (erosiones y sedimentaciones costeras).

No todos los riesgos geológicos tienen la misma peligrosidad; entendiendo ésta como el nivel de gravedad o intensidad con que un fenómeno puede afectar a una región o parte de un territorio ${ }^{6}$. Unos (p. ej. subsidencias) acostumbran a ser lentos y raramente producen víctimas, mientras que otros (p. ej. terremotos) son repentinos y pueden provocar mortandades. Hay riesgos, como los movimientos de ladera, que no suelen acarrear víctimas mortales, pero en cambio su incidencia económica puede ser notable. La frecuencia o probabilidad de los riesgos es también variable. Los hay relativamente infrecuentes, mientras que otros son comunes en determinadas zonas (p. ej. terremotos y erupciones volcánicas en el llamado «cinturón de fuego» del Pacífico).

Un aspecto importante es la concatenación o encadenamiento, en el espacio o en el tiempo, de los riesgos geológicos. A menudo los riesgos no se presentan aislados. Así, las inundaciones suelen ir acompañadas de corrimientos de tierra y erosiones de suelos; ciertos terremotos generan olas gigantes o tsunamis, etc. Eso sin contar las secuelas o efectos que se derivan de ellos: hambrunas, plagas, epidemias...

Conviene, asimismo, diferenciar entre riesgo y catástrofe. Para algunos especialistas, un riesgo natural implicaría sólo una posibilidad de que se produzca daño para el hombre ${ }^{7}$ El término de catástrofe o desastre natural habría que reservarlo, según dichos autores, para aquellos sucesos verdaderamente perjudiciales que ocasionan daños económicos y sociales. De hecho, una catástrofe suele producirse allí donde un riesgo potencial se materializa en condiciones de no prevención, ya sea por la falta de predicción o por la ausencia de medidas. Además, la repercusión de un riesgo geológico no depende únicamente de la naturaleza del mismo, sino de otros factores, tal como la zona geográfica donde se produce. Así, un terremoto en una zona desértica no tiene los efectos catastróficos que tiene un terremoto de similares características en un área densamente poblada. Los efectos tampoco suelen ser los mismos en una ciu-

\footnotetext{
${ }^{4}$ Ayala Carcedo, 1988, pág. 4.

${ }^{5}$ Se entiende por subsidencia el hundimiento gradual, o en ocasiones brusco, de un terreno con poco o nulo movimiento horizontal.

${ }^{6}$ Ayala Carcedo, ibídem., p. 5.

${ }^{7}$ Ayala Carcedo, ibídem., p. 6.
} 
dad en la que predominan las construcciones antisísmicas que en otra que carezca de ellas.

Desastres naturales en el siglo XVIII

De modo similar a otros siglos, en la España del siglo XVIII se vivieron numerosos desastres naturales de diferentes tipos: terremotos, erupciones volcánicas, tsunamis, inundaciones, deslizamientos de tierras... La distribución geográfica de los mismos es desigual. Algunos de estos desastres son recurrentes, sobre todo en ciertas partes del territorio (p. ej. inundaciones en la zona levantina), mientras que otros son más raros o excepcionales (tsumanis) o se hallan restringidos a determinadas áreas geográficas (las erupciones volcánicas en las islas Canarias). Además, hay que que tener en cuenta que el registro documental de dichos sucesos es escaso o incompleto, y que no siempre reflejan su verdadera magnitud, lo que hace difícil o prácticamente imposible la comparación cualitativa y cuantitativa con siglos anteriores o posteriores. En general no puede asegurarse que en el siglo XVIII se hayan producido más o menos desastres naturales que en otras épocas, aunque algunos de ellos pueden incluirse entre los más catastróficos de los tiempos modernos. Para este tipo de cómputos, la perspectiva histórica resulta insuficiente, ya que la medida de tiempo en la que se insertan estos eventos es la escala geológica.

\section{Terremotos}

Un terremoto es uno de los fenómenos geológicos corticales más peligrosos y violentos que puedan darse. Los terremotos se describen en términos de magnitud $^{8}$ e intensidad ${ }^{9}$. Entre los principales terremotos catastróficos, producidos en el mundo en el siglo XVIII, se encuentran los siguientes ${ }^{10}$ :

\footnotetext{
${ }^{8}$ La magnitud es una medida de la energía liberada bruscamente por el terremoto. La escala de Richter expresa el cálculo de dicha energía. Es una escala logarítimica, lo que significa que cada número entero de la escala indica un terremoto que produce una amplitud 10 veces mayor (en la traza de la onda sísmica) que el terremoto descrito con el siguiente dígito inferior. La energía liberada aumenta alrededor de 40 veces entre números enteros. Un terremoto de magnitud 1 representa una energía liberada de alrededor de 170 gramos de TNT, mientras que un seísmo de grado 8 representa la energía equivalente a una explosión de 6 millones de toneladas de TNT. El máximo se situaría en 8,9. (Suárez y Regueiro, 1997, pág. 62).

${ }^{9}$ La intensidad se mide actualmente por la escala de Mercalli modificada, y comprende 12 niveles (de I a XII). Viene a representar los efectos de un seísmo y se basa, entre otros criterios, en la observación de los daños en estructuras hechas por el hombre. No siempre a una gran magnitud corresponde una alta intensidad, y viceversa.

${ }^{10}$ Datos tomados, fundamentalmente, de De Buen, 1896? y Bott, 1987.
} 


\begin{tabular}{cccc} 
Año & Fecha & Lugar & Muertos (aprox.) \\
\hline 1737 & 11 octubre & Calcuta (India) & 300.000 \\
1746 & 28 octubre & Lima (Perú) & 30.000 \\
1755 & 7 junio & Norte de Persia & 40.000 \\
1755 & 1 noviembre & Lisboa (Portugal) & 60.000 \\
1783 & 4 febrero & Calabria (Italia) & 50.000 \\
1797 & 4 febrero & Riobamba (Ecuador) & 40.000 \\
\hline
\end{tabular}

En España, las zonas de mayor riesgo sísmico se concentran principalmente en Andalucía oriental, Murcia y los Pirineos ${ }^{11}$. Entre los temblores de tierra habidos en el siglo XVIII, figuran los siguientes ${ }^{12}$ : Tenerife (24 de diciembre de 1704); Xàtiva (23 de marzo y 2 de abril de 1748); Madrid (31 de marzo de 1761); Granada (18 de noviembre de 1778); La Seu d'Urgell (11 de enero de 1788); Barcelona (16 de julio de 1790); Ejea de los Caballeros (1793).

Ninguno de estos seísmos sobrepasó la intensidad VIII en la escala de Mercalli. Sin embargo, el terremoto que sí fue sentido en toda la Península Ibérica, con una intensidad estimada de IX-X según los lugares, fue el de Lisboa de 1755. Dicho terremoto fue el que más impacto causó no sólo en la Península Ibérica, sino en toda Europa, y el que en su momento generó una mayor cantidad de documentación, gracias a la cual las circunstancias que lo envolvieron son hoy en día bastante bien conocidas.

\section{El terremoto de Lisboa de 1755}

A partir de 1750, gran parte de la Europa occidental se vio repetidamente afectada por terremotos de distinta intensidad. En la mañana del día 1 de noviembre de 1755 (festividad de Todos los Santos), en Lisboa, a las nueve y treinta y cinco de la mañana, cuando la mayoría de sus habitantes asistían a misa en las iglesias, una terrible sacudida estremeció la ciudad. Era el inicio de uno de los más impresionantes y calamitosos terremotos que registra la historia. No hubo signos externos que lo precedieran, tampoco ruidos premonitorios (retumbos). La sorpresa fue total. Al principio fueron unas rápidas y pequeñas vibraciones; medio minuto después se produjeron unos movimientos rápidos y violentos que duraron dos minutos. Un minuto más tarde siguió un fuerte movimiento ascendente que finalizó al cabo de dos minutos y medio. En unos cinco minutos quedó gran parte de la capital prácticamente reducida

\footnotetext{
1983.

${ }^{11}$ Para un estudio general de la sismicidad en el área ibérica véase Mezcua y Martínez Solares,

12 Datos tomados, básicamente, de los periódicos Mercurio Histórico y Político (periodo 1757-1783) y Memorial Literario, Instructivo y Curioso de la Corte de Madrid (periodo 1787-1790).
} 
a escombros. Se calcula que perecieron unos 60.000 habitantes de los 275.000 que tenía Lisboa ${ }^{13}$.

Dado que el epicentro del terremoto se situó en el Atlántico, a unos 100 $\mathrm{km}$ al este de Lisboa, éste vino acompañado de un tren de impresionantes olas de marea o tsunamis, que inundaron y barrieron la parte baja de la ciudad. Algunas de estas gigantescas olas alcanzaron los 27 metros de altura. Consecuentemente, las aguas del río Tajo subieron bruscamente varios metros. En algunos sitios de la costa portuguesa el nivel del mar se elevó por encima de los quince metros. Durante veinticuatro horas el mar permaneció en un estado de constante agitación. Se considera al maremoto de Lisboa el más violento generado en el Atlántico en tiempos históricos. (El período de retorno ${ }^{14}$ de un fenómeno de estas características se sitúa en torno a 1000 años).

Otros tsunamis menos intensos se sucedieron en los meses siguientes en las costas mediterránreas y atlánticas, principalmente en la portuguesa y española. Al otro lado del Atlántico (Antillas y Norteamérica) igualmente llegaron las olas de marea.

El área geográfica en la que se sintió el terremoto fue muy extensa. Afectó con intensidad destructiva todo Portugal, España y la región noroccidental de Africa. De hecho se percibió en mayor o menor grado en todos los países de la Europa central y septentrional, en los cuales se produjeron seiches ${ }^{15}$ y se alteraron los flujos de los manantiales. Se estima que la magnitud del terremoto de Lisboa fue de 8,7 en la escala de Richter; y su intensidad, a tenor de los efectos registrados, la máxima de XII grados en la escala de Mercalli modificada. Las réplicas posteriores fueron muy numerosas y la zona lisboeta estuvo afectada por temblores de tierra de diversa intensidad durante cinco años, lo que retrasó considerablemente las labores de reconstrucción de la ciudad.

En España el terremoto causó gran alarma y cuantiosos daños, sobre todo en la zona sureste, provocando el derrumbe de casas y edificios. Muchos momumentos históricos de dicha zona (especialmente campanarios y torres de iglesias) todavía muestran las grietas producidas por el seísmo. El tsunami que le siguió alcanzó de lleno la bahía de Cádiz, causando graves daños en toda la costa y un millar de muertos ${ }^{16}$. El cercano pueblo de Conil quedó prácticamente des-

\footnotetext{
${ }^{13}$ Sobre el terremoto de Lisboa hay abundante bibliografía. Entre otros trabajos pueden consultarse los de Capel, 1980 y Ordaz, 1983.

${ }^{14} \mathrm{El}$ "periodo de retorno" indica el tiempo en que se puede producir un suceso o riesgo de un determinado nivel o intensidad.

${ }^{15}$ Oscilación anormal del nivel de las aguas de una cuenca cerrada o semicerrada (bahía, lago, etc.).

${ }^{16}$ La ciudad de Cádiz ya había sufrido otro tsunami en 1731, cuando se retiró el mar una legua, dejando al descubierto, en Santi-Petri, los restos de la antigua Gades y el templo de Hércules. (Dato de M. Catalán, en Ayala Carcedo y Elizaga Muñoz, 1987, pág. 26)
} 
truido. También afectó a lugares tan distantes entre sí como Galicia, Melilla e islas Canarias.

El terremoto de Lisboa motivó grandes polémicas en los medios sociales y científicos de Europa. En España también se produjeron reacciones. A este respecto pueden diferenciarse dos grandes grupos de opinión: por un lado el de aquellos autores que ven el terremoto como un «castigo de Dios» por los pecados y desórdenes de la humanidad y, en consecuencia, no contemplan otra causa que la divina o moral; y por otro lado, el de aquellos que, sin poner en duda los designios del Supremo Hacedor como causa última, analizan el terremoto desde el punto de vista de su causa física inmediata.

Dentro de este último grupo, las opiniones fueron encontradas, y la polémica se agudizó entre los partidarios de las ideas tradicionales o aristotélicas sobre el origen de los terremotos, y los que planteaban una revisión más o menos profunda de las mismas, sirviéndose de otras hipótesis más modernas, como las químicas, eléctricas o teluro-mecánicas.

Entre los que, desde el primer momento, abogaron por una causa física del terremoto estuvo el P. Feijoo, quien escribió una serie de cartas entre noviembre de 1755 y enero de 1756, las cuales fueron publicadas poco tiempo después por D. José Luis Roche, comerciante y gran amigo del Padre Maestro $^{17}$. La edición de las cartas de Feijoo avivó notablemente la polémica y provocó la respuesta inmediata de los partidarios aristotélicos, como fray Miguel Cabrera, Ortiz Gallardo o Nifo, quienes siguiendo la teoría de los «pirofila$\operatorname{cios}{ }^{18}$ representaban las tesis más comúnmente aceptadas en aquellos momentos. Entre los que se pusieron de parte del benedictino están el citado Roche o el P. Cevallos. En líneas generales puede decirse que el terremoto de Lisboa y el también catastrófico de Calabria de 1783 sirvieron para suscitar la atención de los científicos y dar un impulso definitivo a los estudios sismológicos.

\section{Erupciones volcánicas}

La erupción de un volcán es uno de los fenómenos naturales más vistosos y llamativos, pero es también uno los riesgos naturales más destructivos, no sólo por la acción directa de la erupción (flujos de lava, material piroclástico, nubes de gases) sino porque, en ocasiones, viene acompañada de una concate-

\footnotetext{
${ }^{17}$ Las cartas se publicaron con el título Nuevo systhema sobre la causa physica de los Terremotos, explicado por los phenomenos eléctricos, y adaptado al que padeció España en primero de Noviembre del año antecedente de 1755 (Imprenta de la Casa Real de las Cadenas, Puerto de Santa María, 1756).

${ }^{18}$ Expuesta por Athanasius Kircher en Mundus subterraneus (Roma, 1675). Véase Capel, 1980.
} 
nación de desastres (cambios climáticos, terremotos, etc.) de efectos devastadores $^{19}$.

En el siglo XVIII se produjeron numerosas explosiones volcánicas de calamitosas consecuencias. Por ejemplo, en la segunda mitad del siglo entraron en erupción, entre otros, conocidos volcanes activos como el Taal (Filipinas, 1754), Etna y Vesubio (Italia, 1757), Jorullo (México, 1759), Unzen (Japón, 1792), Papandayang (Java, 1772), Kilauea (Hawai, 1789) o Hekla (Islandia, 1789). Sólo en el año 1766 explosionaron violentamente Cotopaxi (Ecuador), Mayón (Filipinas), Etna (Sicilia) y Vesubio (Italia). Dos de las erupciones más importantes, por sus consecuencias, tuvieron lugar en $1783^{20}$ :

- Laki (Islandia). Una de las mayores erupciones de flujo de lava que se hayan visto jamás. Una fisura de $20 \mathrm{~km}$ vertió lava durante ocho meses, formando una capa de 30 metros que cubrió una superficie de unos 900 kilómetros cuadrados. Destruyó las cosechas, diezmó el ganado y derivó en hambruna y enfermedades. Murieron unas 9000 personas. La emisión de gran cantidad de gases y polvo contaminó la atmósfera durante años, afectó a los patrones de crecimiento de las plantas, e indujo cambios climáticos en Europa, con descensos de temperaturas y contaminación atmosférica que dio lugar a crepúsculos de llamativos colores ${ }^{21}$.

- Asama (Japón). Gran devastación por flujos piroclásticos. A causa de la explosión cedió la pared del cráter, provocando el vertido de alrededor de 1 km cúbico de lava, cenizas y lodo. Derivó también en cambios climáticos nefastos, con un encadenamiento de desastres — sequía, seguida de inundacionesque ocasionó en total cerca de un millón de muertos ( «hambruna de Ten-mei») ${ }^{22}$.

${ }^{19}$ La fuerza o energía liberada por una explosión volcánica se mide mediante el «Índice de Explosividad Volcánica» (VEI). Este índice, propuesto por C. G. Newhall, del U. S. Geological Survey, se basa en la estimación del volumen de material (rocas y cenizas) expulsado por el volcán, la altura de la nube y otras observaciones. La escala comprende 8 niveles, siendo el 1 una explosión muy pequeña y el 5 muy grande. Ninguna explosión en los últimos 10.000 años se le ha asignado un índice VEI de 8 (SuÁrez y Regueiro, ibídem, pág. 83).

${ }^{20}$ MiLne, ibídem, pág. 127.

${ }^{21}$ De esta erupción se hizo eco, aunque con retraso, el Mercurio de España (julio de 1784, págs. 252 253).

22 Próxima en el tiempo, aunque fuera ya del siglo xvIII propiamente dicho, habría que mencionar también la erupción en 1815 del volcán Tambora (Indonesia), considerada la mayor explosión volcánica de los tiempos históricos (VEI 7, equivalente a unas 10.000 bombas atómicas). Se expulsaron 147 kilómetros cúbicos de material y causó más de 90.000 muertos. La columna de erupción alcanzó los 43 kilómetros de altura, la más alta registrada hasta el momento. La enorme cantidad de cenizas y aerosoles gaseosos (especialmente de dióxido de azufre) evacuados a la estratosfera provocó cambios climáticos apreciables en Europa y América, rebajando significativamente la insolación y la temperatura media del planeta $\left(1,1^{\circ} \mathrm{C}\right.$ durante dos años). 1816 se conoció como " el año sin verano». Hubo nevadas en julio y agosto en algunos sitios de Europa y Norteamérica y se perdieron muchas cosechas. (Sigurdsson, ibídem, págs. 10-11). 
Comparadas con estas colosales erupciones, las sucedidas en España, en el siglo XVIII son de mucho menor rango y consecuencias. Lógicamente, todas las erupciones tuvieron lugar en el archipiélago canario, dado que en la Península no existen volcanes activos ${ }^{23}$. En el cuadro adjunto se relacionan las erupciones históricas habidas en las islas Canarias en el siglo XVIII ${ }^{24}$.

\begin{tabular}{cccc} 
Isla & Fecha & Nombre del volcán & Duración (en días) \\
\hline Tenerife & 1704 & Siete Fuentes & - \\
Tenerife & 1705 & Fasnia & - \\
Tenerife & $2-\mathrm{II}-1705$ & Montaña Arenas & 24 \\
Tenerife & $5-\mathrm{V}-1706$ & Montaña Negra & 9 \\
La Palma & $9-\mathrm{X}-1712$ & El Charco & 56 \\
Lanzarote & $1-\mathrm{IX}-1730$ & Timanfaya & 6 años \\
Tenerife & $9-\mathrm{VI}-1798$ & Chahorra & 92 \\
Hierro & $?-\mathrm{V}-1793$ & Lomo Negro & $15 ?$
\end{tabular}

Como puede apreciarse, más de la mitad de las erupciones registradas se localizan en Tenerife. Es de destacar que casi todas las erupciones fueron de tipo explosivo, de baja intensidad y corta duración (de días a meses), surgiendo un pequeño cono y coladas lávicas de escaso recorrido. Muy pocas supusieron un grave peligro a la población, dando tiempo suficiente para su evacuación. La más importante, tanto por su duración (seis años), el volumen de materiales expelido (unos 700 millones de metros cúbicos) y el área cubierta por los materiales eyectados (alrededor de 150 milllones de metros cuadrados) fue la de Timanfaya, en Lanzarote, ocurrida en septiembre de $1730^{25}$. Las lluvias de piroclastos y las lavas arrojadas de varias fisuras del terreno se prolongaron varios años, enterrando tierras de labor fértiles y ocasionando la emigración forzosa de un $70 \%$ de la población afectada. No hubo víctimas humanas, aunque sí animales debido a las emanaciones de gases.

\section{Inundaciones}

Las inundaciones son riesgos geoclimáticos que se repiten cada cierto tiempo en la mayoría de sistemas fluviales importantes. Se producen básicamente cuando las aguas procedentes de precipitaciones o del deshielo de las nieves se dirigen hacia un cauce de orden mayor desde su cuenca de recepción.

\footnotetext{
${ }^{23}$ Véase Romero et al., 1986.

${ }^{24}$ En Suárez y Regueiro, ibídem, pág. 90.

${ }^{25}$ Consúltese, para esta erupción, Romero Ruz, 1997.
} 
Así pues, las mayores inundaciones tienen lugar, bien a partir de deshielos súbitos, fuera de la temporada usual; o bien a causa de lluvias torrenciales sobre un área amplia (caso de huracanes y tifones) o más reducida (chubascos y tormentas). Las consecuencias catastróficas de una inundación varían mucho, según la intensidad de la misma y la población y terrenos afectados. Algunos de los desastres naturales con mayor número de víctimas mortales, además de graves pérdidas económicas, se deben a inundaciones ${ }^{26}$.

En España las inundaciones son frecuentes en la zona mediterránea: Cataluña, Comunidad Valenciana, Murcia y Andalucía oriental. También son relativamente frecuentes en los Pirineos, la cornisa cantábrica y en la zona centro. Muchas de estas inundaciones se deben al fenómeno de la «gota fría»; es decir, a fuertes precipitaciones en forma de lluvia, de carácter estacional (generalmente al inicio de la primavera y el otoño) y de corta duración. Son especialmente peligrosas las rieras y ramblas mediterráneas, cauces secos la mayor parte del año, capaces de transformarse, en unos pocos minutos, en avenidas de agua, lodo y piedras (inundación de tipo «relámpago»o flash) ${ }^{27}$.

En el setecientos se produjeron en España inundaciones de diversa intensidad, especialmente en la zona levantina. Entre ellas cabe citar la del río Turia, en Valencia, el 16 de septiembre de 1731; y la riada del Ebro a su paso por Tortosa y pueblos ribereños, el 8 de octubre de 1787, que causó 183 muertos y se llevó por delante más de mil casas. También en el interior del país hubo importantes crecidas que provocaron estragos en vidas y haciendas, siendo de destacar las de Sangüesa, Estella y Pamplona en otoño de 1787. Al año siguiente se desbordaron, entre otros, el Esgueva en Valladolid (febrero) y el Duero en Tordesillas (marzo).

Hay que señalar que en la época de que se trata, y aún hasta mucho tiempo después, no había medidas estructurales de prevención (presas, canalizaciones de ríos a su paso por núcleos urbanos, etc) o éstas eran muy escasas e insuficientes. Tampoco existían, como en la actualidad, servicios de predicción meteorológica que alertasen a la población de posibles riadas; por lo que los efectos destructivos de las mismas solían resultar casi siempre catastróficos, aunque la densidad de población afectada fuese bastante menor que en la actualidad.

${ }^{26}$ Así, por ejemplo, los ríos Yangtze y Amarillo, en China, muestran periódicamente grandes avenidas y desbordamientos que afectan a cientos de miles de personas. El río Amarillo, conocido como el «dolor de China», ha causado a lo largo de la historia más víctimas mortales y damnificados que ningún otro desastre natural. Por citar un solo suceso: Como consecuencia de la inundación del año 1887 se calcula que se produjeron entre uno y seis millones de víctimas mortales (MILNE, ibídem, págs. 92-93). Véase, también, MiLleR y MiLler, 2000.

${ }^{27}$ Suárez y Regueiro, ibídem, pág. 151. 
Por otro lado, al considerar la frecuencia de inundaciones en el setecientos hay que tener en cuenta, tratándose de un fenómeno en el que el clima es determinante, que esta época se sitúa dentro de la llamada «pequeña edad de hielo» o «pequeña glaciación», que abarca grosso modo desde el siglo xv hasta mediados del xIx, con el mínimo térmico comprendido entre 1570 y 1730, aproximadamente ${ }^{28}$. Dicho episodio se caracteriza por un enfriamiento generalizado de la atmósfera de la Tierra, en torno a $0,5^{\circ} \mathrm{C}$, lo que ocasionó cambios climáticos variables (por ejemplo el número de tormentas en el Mar del Norte disminuyó sensiblemente), así como graves repercusiones sociales y económicas en muchos países del hemisferio norte ${ }^{29}$.

\section{Movimientos de ladera}

En este grupo se incluyen los corrimientos de tierra o deslizamientos, así como los aludes de nieve y hielo. Los deslizamientos se definen como movimientos rápidos de una masa de terreno (suelo y rocas) por fuerzas gravitatorias ${ }^{30}$. El volumen de masa es muy variable y depende de la naturaleza y condiciones del terreno, así como de factores externos (lluvias, inundaciones, terremotos, obras de construcción, etc) que actúan como factores desencadenantes.

Algunos de los aludes o grandes deslizamientos conocidos han movido varios kilómetros cúbicos de material y acarreado miles de víctimas y cuantiosas pérdidas materiales ${ }^{31}$. En España, la geología, el relieve y las condiciones climáticas, favorecen la aparición de movimientos de ladera. Estos se localizan principalmente en las zonas montañosas del norte y sur de la Península, donde suelen estar asociados a la topografía y a la naturaleza arenosa y arcillosa de los materiales geológicos. Los deslizamientos son frecuentes en los Pirineos, cordilleras Cantábrica y Bética y ciertas áreas del Macizo Ibérico. En Asturias son muy conocidos, y no menos temidos, los corrimientos de tierra («argayos») en materiales rocosos fracturados y meteorizados, sobre todo después de fuertes lluvias.

A lo largo del siglo XVIII hubo numerosos deslizamientos, si bien muchos de ellos no alcanzan la categoría de catastróficos ni se hallan debidamente documentados, por lo que resulta difícil hacer una valoración de los mismos. Entre los más significativos desastres de este tipo se pueden citar: el de Inza (Nava-

${ }^{28}$ Véase Vázquez Abeledo, 1998, págs. 273-274; Kump et al., 1999, págs. 236-237.

29 Bryant, 1997, págs. 90-91.

${ }^{30}$ SuÁrez y Regueiro, ibídem, pag. 97-111.

${ }^{31}$ A este respecto, los aludes de los Alpes son tristemente famosos: han diezmado a ejércitos como el de Aníbal (280 a. de C.) o a los de Italia y Austria en la Primera Guerra Mundial, con cifras en torno a los 18.000 y 10.000 muertos, respectivamente (Suárez y Regueiro, ibídem, pág. 98). 
rra), en 1714, que destruyó gran parte del pueblo; el de Benamají (Córdoba), en 1739, que derribó varias casas; y los de Biniarroi (Mallorca), en 1721 y en 1783, que produjeron también importantes destrozos ${ }^{32}$.

\section{Desastres naturales y catastrofismo}

A nivel popular, y a lo largo del siglo, los desastres naturales son contemplados por muchas personas como sucesos ominosos, de difícil explicación. Al temor lógico por los efectos producidos se une su ineluctabilidad y la ignorancia de sus causas; lo que hace, si cabe, más terribles y amenazadores dichos sucesos. Esta percepción fatalista y oscura de los desastres naturales empieza a cambiar sobre todo a finales del siglo xVIII, a medida que se avanza en la comprensión de la historia y el funcionamiento de la Tierra, proporcionando explicaciones racionales para estos fenómenos naturales y despojándolos de connotaciones extracientíficas.

Con el nacimiento de la geología moderna, dos modelos o líneas maestras de pensamiento pugnan por interpretar científicamente los fenómenos geológicos. De forma muy simplificada tenemos, por un lado, el llamado catastrofismo, que pretende explicar la historia geológica de la Tierra mediante la sucesión de grandes cataclismos, los cuales suponen grandes saltos cualitativos y cuantitativos. El último de éstos habría sido el Diluvio Universal. Se trata de un modelo discontinuo, con bruscos cortes y rupturas. En esta línea dominante tendríamos que incluir, como precedentes, la mayoría de las «teorías de la Tierra» de Burnet, Whiston, Ray, etc.— que ven la luz a finales del siglo xvI y principios del xvIII. Es también el modelo propugnado por Georges Cuvier en su Discurso sobre las revoluciones del Globo (1812). Por otro lado tenemos el gradualismo, que presupone una evolución suave y continua de la dinámica terrestre, sin bruscos cortes o rupturas violentas, de la que no se excluyen las catástrofes tal como se entienden hoy en día. Dicha modalidad es la que adopta James Hutton en su Teoría de la Tierra $(1776)^{33}$.

En esta oposición catastrofismo vs. gradualismo, la tesis de Hutton marca un hito importante dado que introduce un giro significativo y matizaciones que rompen conceptualmente con arraigadas ideas de épocas precedentes. Hasta este momento la creencia dominante era que las grandes cataclismos del pasado eran fenómenos extraordinarios, que obedecían en última instancia a dictados divi-

\footnotetext{
${ }^{32}$ Suárez y Regueiro, ibídem, pág. 107.

${ }^{33}$ Para el debate entre catastrofistas y gradualistas consúltese Hallam, 1985.
} 
nos o a causas «no naturales», es decir, preternaturales. Así lo entendían algunos diluvialistas, para quienes los fósiles encontrados en las montañas sólo se podían explicar a partir de la gran inundación bíblica. Hutton, en cambio, considera las catástrofes como fenómenos meramente geológicos, debidos a causas exclusivamente naturales, y las inserta dentro de un modelo de evolución actualista, en el que los mismos procesos que actúan hoy en día en la superficie terrestre son los que han actuado en el pasado y que acturán en el futuro ${ }^{34}$. Sin embargo, este modelo no adquirió carta de naturaleza hasta bien entrado el siglo xIx, merced, sobre todo, a las ideas uniformitaristas contenidas en los Principios de Geología (1830-1833), de Charles Lyell.

\section{Bibliografía}

Ayala Carcedo, Francisco Javier y Elizaga Muñoz, Emilio (eds.). Impacto económico y social de los riesgos geológicos en España. Instituto Geológico y Minero de España, Madrid, 1987.

Ayala Carcedo, Francisco Javier. «Introducción a los riesgos geológicos». En: Riesgos Geológicos, Instituto Geológico y Minero de España, Madrid, 1988, p. 3-13.

Bott, Bruce A. Terremotos. Ediciones Orbis, S.A., Barcelona, 1987.

Bryant, Edward. Climate, Process \& Change. Cambridge University Press, 1997.

CAPEL, Horacio. «Organicismo, fuego interior y terremotos en la ciencia española del siglo XVIII». GeoCrítica, Ediciones de la Universidad de Barcelona, 1976.

Davis, Lee. Natural Disaster: From the Black Plague to the Eruption of Mt. Pinatubo. Facts on File, Nueva York, 1991.

DE BuEn, Odón. «Temblores de tierra». En: Historia Natural. T. II, Manuel Soler, Barcelona, s.f. (1896?).

Galanopoulos, Angelos George y Bacon, Edward. Atlantis. The Truth Behind the Legend. Bobbs-Merrill Co., Nueva York, 1969.

Hallam, Arthur. Grandes controversias geológicas. Labor, Barcelona, 1985.

Kump, Lee R., Kasting, James F. y Crane, Robert G. The Earth System. Prentice Hall, New Jersey, 1999.

Mezcua, J. y Martínez Solares, J.M. Sismicidad del Área Ibero-Mogrebí. Public. Tec. nº. 203, Instituto Geográfico Nacional, Madrid, 1983.

Miller, E. Willard y Miller, Ruby M. Natural Disasters: Floods. Contemporary World Issues, Santa Barbara, 2000.

\footnotetext{
${ }^{34}$ La implicación de los riesgos geológicos en el dilema actualismo-catastrofismo es tratado por PEDRAZA Gilsanz, 1988.
} 
MiLne, Antony. Floodshock. The Drowning of Planet Earth. Alan Sutton, Gloucester, 1986. Milne, Antony. Doomsday. The Science of Catastrophic Events. Praeger, Londres, 2000.

Ordaz, Jorge. «El terremoto de Lisboa de 1755 y su impacto en el ámbito científico español». II Simposio sobre el P. Feijoo y su época (Ponencias y Comunicaciones), Oviedo, T. II, 1983, pp. 433-442.

Pedraza Gilsanz, Javier. «Los riesgos geológicos a la luz del dilema actualismo-catastrofismo». En: Riesgos Geológicos. Instituto Geológico y Minero de España, Madrid, 1988, pp. 37-44.

Romero Ruz, C. Crónicas documentales sobre las erupciones de Lanzarote. Fundación César Manrique, Colección Torcusa, 3, Teguise (Lanzarote), 1997.

Romero, Carmen, Quirantes, Francisco y Martínez de Pisón, Eduardo. Los volcanes. Guía física de España 1. Alianza Editorial, Madrid, 1986.

Suárez. Luis y Regueiro, Manuel (eds.). Guía ciudadana de los riesgos geológicos. Ilustre Colegio Oficial de Geólogos de España, Madrid, 1997.

Sigurdsson, Haraldur (ed.). Encyclopedia of Volcanoes. Academic Press, New York, 2000.

Vázquez Abeledo, Manuel. La historia del sol y el cambio climático. McGraw Hill, Madrid, 1998. 